\title{
MIXED AND DIRECTIONAL DERIVATIVES
}

\author{
W. CHEN AND Z. DITZIAN
}

(Communicated by William J. Davis)

Abstract. The estimate

$$
\left\|\frac{\partial^{k} f(x)}{\partial \xi_{1} \cdots \partial \xi_{k}}\right\| \leq \sup _{\xi}\left\|\frac{\partial^{k} f(x)}{\partial \xi^{k}}\right\|
$$

is proved for various spaces of functions over domains in $R^{d}$, where $\partial f / \partial \xi_{i}$ is the directional derivative of $f$ in the $\xi_{i}$ direction and $\xi_{1}, \ldots, \xi_{k}$ are any $k$ directions.

\section{INTRODUCTION}

Estimates of mixed derivatives by directional derivatives were proved (implicitly) in the effort to characterize the $K$-functional of the pair $\left(L_{p}, W_{p}^{r}\right)$ (see [1, Ch. 5]). Though this fact is not stated explicitly, the Kemperman Lemma (see [1, Lemma 4.11, p. 338]) actually implies for a domain $D$ which is nice enough

$$
\left\|\frac{\partial^{k} f}{\partial \xi_{1} \cdots \partial \xi_{k}}\right\|_{L_{p}(D)} \leq C(k) \sup _{\xi}\left\|\frac{\partial^{k} f}{\partial \xi^{k}}\right\|_{L_{p}(D)}
$$

with $C(k)$ that increases geometrically with $k$ (see [1, Ch. 5]). In this paper we will show that $C(k)$ can be replaced by 1 , which is obviously the best possible constant, as the directions $\xi_{1}, \ldots, \xi_{k}$ are any $k$ directions, or unit vectors, in $R^{d}$. We remark also that here the assumption that $D$ is open in $R^{d}$ is sufficient. Furthermore, the result is valid for many other spaces, as will be shown in $\S 5$. We hope that this inequality will help in settling other problems, for example the investigation of the still open problem of best constant for the multivariate Landau-Kolmogorov inequality (see [2]). We further believe that (1.1) with the elegant best constant $C(k)=1$ is desirable by itself.

\section{THE LOCAL RESUlT}

The local version of the result of this paper is given in the following theorem, which is essentially the basis for all other results of the paper.

Received by the editors February 14, 1989 and, in revised form, May 1, 1989.

1980 Mathematics Subject Classification (1985 Revision). Primary 26D10; Secondary 26B05.

The second author was supported by NSERC A4816. 
Theorem 2.1. Suppose $\left(\partial^{k} / \partial \xi^{k}\right) f(x)$ exists and is continuous in a neighbourhood $U \subset R^{d}$ of $x_{0}$ for any direction $\xi$. Then the mixed derivative $\left(\partial^{k} /\left(\partial \xi_{1} \cdots \partial \xi_{k}\right)\right) f(x)$ exists, is continuous in a neighbourhood of $x_{0}$, and

$$
\left|\frac{\partial}{\partial \xi_{1}} \cdots \frac{\partial}{\partial \xi_{k}} f\left(x_{0}\right)\right| \leq \sup _{\xi}\left|\frac{\partial^{k}}{\partial \xi^{k}} f\left(x_{0}\right)\right| .
$$

Remark. We note that $\xi_{i}$ are any $k$ directions in $R^{d}$.

We observe that Theorem 2.1 implies for an open domain $D$ and $\left(\partial^{k} / \partial \xi^{k}\right) f(x) \in C(D)$ that

$$
\frac{\partial}{\partial \xi_{1}} \cdots \frac{\partial}{\partial \xi_{k}} f(x) \in C(D)
$$

and

$$
\left\|\frac{\partial}{\partial \xi_{1}} \cdots \frac{\partial}{\partial \xi_{k}} f(x)\right\|_{C(D)} \leq \sup _{\xi}\left\|\left(\frac{\partial}{\partial \xi}\right)^{k} f(x)\right\|_{C(D)} .
$$

We will actually prove the result for the spaces of functions $L_{p}(D)$ and $C(D)$ simultaneously.

Theorem 2.2. Suppose $f \in B,\left(\partial^{k} / \partial \xi^{k}\right) f \in B$ for all $\xi$ and $\left\|\partial^{k} f / \partial \xi^{k}\right\|_{B} \leq M$, where either $B=L_{p}(D) \quad 1 \leq p<\infty$ or $B=C(D)$, $D$ being an open set in $R^{d}$. Then

$$
\frac{\partial}{\partial \xi_{1}} \cdots \frac{\partial}{\partial \xi_{k}} f \in B
$$

and

$$
\left\|\frac{\partial}{\partial \xi_{1}} \cdots \frac{\partial}{\partial \xi_{k}} f(x)\right\|_{B} \leq \sup _{\xi}\left\|\left(\frac{\partial}{\partial \xi}\right)^{k} f(x)\right\|_{B} .
$$

Remark 2.3. It sufficient to prove that

$$
\frac{\partial}{\partial \xi_{1}} \cdots \frac{\partial}{\partial \xi_{k}} f \in B_{\varepsilon}
$$

and

$$
\left\|\frac{\partial}{\partial \xi_{1}} \cdots \frac{\partial}{\partial \xi_{k}} f(x)\right\|_{B_{\varepsilon}} \leq \sup _{\xi}\left\|\frac{\partial^{k}}{\partial \xi^{k}} f(x)\right\|_{B}
$$

where $B_{\varepsilon}=L_{p}\left(D_{\varepsilon}\right)$ or $B_{\varepsilon}=C\left(D_{\varepsilon}\right)$ and where

$$
D_{\varepsilon} \equiv\{x ; x+B(\varepsilon) \subset D\}, \quad B(\varepsilon) \equiv\{y ;\|y\| \leq \varepsilon\}
$$

and $\|y\|$ is the Euclidean norm in $R^{d}$. That the inequality (2.3) implies (2.4) is clear for $B=C(D)$ and follows from the theorem on monotone convergence for $B=L_{p}(D)$. 
For the proof which will be carried through in $\S 4$ using several lemmas stated and proved in $\S 3$, we need the following concepts. For a function space on domain $D, S(D)$ we define the transformations

$$
T(y) f(x)=f(x+y), \quad T(y): S(D) \rightarrow S(D-y)
$$

and

$$
\Delta_{y} f(x)=(T(y)-I) f(x)=f(x+y)-f(x), \quad \Delta_{y}: S(D) \rightarrow S(D \cap(D-y)) .
$$

In the next section, and when proving Theorem $2.2, S(D)$ will be $L_{p}\left(D_{*}\right)$ or $C\left(D_{*}\right)$ where $D_{*}$ would be $D_{\varepsilon}$ and its translates. We give (2.6) and (2.7) in the present generality to accommodate some remarks in $\S 5$.

\section{SOME CRUCIAL LEMMAS}

We first prove the following equivalence result.

Lemma 3.1. Suppose $f \in L_{p}(D)$ or $f \in C(D)$. Then the derivative

$$
\frac{\partial}{\partial \xi_{1}} \cdots \frac{\partial}{\partial \xi_{k}} f(x)
$$

exists in $L_{p}(D)$ or $C(D)$ as a local strong derivative if and only if

$$
h^{-k} \Delta_{h \xi_{1}} \cdots \Delta_{h \xi_{k}} f(x) \rightarrow g(x) \text { in } L_{p}\left(D_{\varepsilon}\right) \text { or } C\left(D_{\varepsilon}\right)
$$

for every $\varepsilon>0$. In this case

$$
g(x)=\frac{\partial}{\partial \xi_{1}} \cdots \frac{\partial}{\partial \xi_{k}} f(x)
$$

a.e. in $D_{\varepsilon}$ for $B=L_{p}(D)$ and everywhere in $D_{\varepsilon}$ for $B=C(D)$.

Remark. For $h$ small enough, which depends on $\varepsilon, \Delta_{h \xi_{1}} \cdots \Delta_{h \xi_{k}}$ is defined on $L_{p}\left(D_{\varepsilon}\right)$.

Proof. By the repeated application of the mean value theorem, we see that the first assumption implies the second for the space of continuous functions. For the space $L_{p}$ the same argument is used essentially, and we write for $|h| \leq \varepsilon / k$, $x \in D_{\varepsilon}$

$$
\begin{gathered}
h^{-k} \Delta_{h \xi_{1}} \cdots \Delta_{h \xi_{k}} f(x) \\
=h^{-k} \int_{0}^{h} T\left(u_{k} \xi_{k}\right) \int_{0}^{h} \cdots \int_{0}^{h} T\left(u_{1} \xi_{1}\right) \frac{\partial^{k}}{\partial \xi_{1} \cdots \partial \xi_{k}} f(x) d u_{1} \cdots d u_{k} \text { a.e. }
\end{gathered}
$$

Obviously, the operator $O_{h}: L_{p}(D) \rightarrow L_{p}\left(D_{\varepsilon}\right)$ given by

$$
O_{h} \equiv h^{-k} \int_{0}^{h} T\left(u_{k} \xi_{k}\right) \int_{0}^{h} \cdots \int_{0}^{h} T\left(u_{1} \xi_{1}\right) d u_{1} \cdots d u_{k}
$$

is bounded, and

$$
\left\|O_{h} \varphi-\varphi\right\|_{L_{p}\left(D_{\varepsilon}\right)} \rightarrow 0 \quad \text { for all } \varphi \in L_{p}(D) .
$$


We now show that for $g(x)$ given by (3.1) and $\left|t_{i}\right|<\varepsilon / k$

$$
\begin{aligned}
& \frac{1}{t_{1} \cdots t_{k}} \Delta_{t_{1} \xi_{1}} \cdots \Delta_{t_{k} \xi_{k}} f(x) \\
& =\frac{1}{t_{1} \cdots t_{k}} \int_{0}^{t_{k}} T\left(u_{k} \xi_{k}\right) \int_{0}^{t_{k-1}} \cdots \int_{0}^{t_{1}} T\left(u_{1} \xi_{1}\right) g(x) d u_{1} \cdots d u_{k}
\end{aligned}
$$

a.e. in $D_{2 \varepsilon}$ when $g$ in $(3.1)$ is in $L_{p}\left(D_{\varepsilon}\right) \quad 1 \leq p<\infty$, and everywhere in $D_{2 \varepsilon}$ when $g \in C\left(D_{\varepsilon}\right)$. To show (3.3) we observe that for $\left|t_{i}\right|<\varepsilon / k$

$$
O_{t_{k} \cdots t_{k}} \equiv \frac{1}{t_{1} \cdots t_{k}} \int_{0}^{t_{k}} T\left(u_{k} \xi_{k}\right) \cdots \int_{0}^{t_{1}} T\left(u_{1} \xi_{1}\right) d u_{1} \cdots d u_{k}
$$

is a bounded transformation from $L_{p}\left(D_{\varepsilon}\right)$ to $L_{p}\left(D_{2 \varepsilon}\right) \quad(1 \leq p<\infty)$ or from $C\left(D_{\varepsilon}\right)$ to $C\left(D_{2 \varepsilon}\right)$. In fact for $t_{i}$ small enough

$$
\left\|O_{t_{1} \cdots t_{k}} \varphi\right\|_{B_{\varepsilon}} \leq\|\varphi\|_{B}
$$

and

$$
\lim _{t_{i} \rightarrow 0}\left\|O_{t_{1} \cdots t_{k}} \varphi(x)-\varphi(x)\right\|_{B_{\varepsilon}}=0,
$$

no matter what order of $t_{i}$ we use in the limit. This implies

$$
\begin{aligned}
O_{t_{1} \cdots t_{k}} g(x) & =\lim _{h \rightarrow 0} h^{-k} O_{t_{1} \cdots t_{k}} \Delta_{h \xi_{1}} \cdots \Delta_{h \xi_{k}} f(x) \\
& =\lim _{h \rightarrow 0} O_{h, \ldots, h}\left\{\frac{1}{t_{1} \cdots t_{k}} \Delta_{t_{1} \xi_{1}} \cdots \Delta_{t_{k} \xi_{k}} f(x)\right\} \\
& =\frac{1}{t_{1} \cdots t_{k}} \Delta_{t_{1} \xi_{1}} \cdots \Delta_{t_{k} \xi_{k}} f(x)
\end{aligned}
$$

for $x \in D_{2 \varepsilon}$. We now write

$$
\begin{aligned}
g(x) & =\lim _{t_{1} \rightarrow 0} \cdots \lim _{t_{k} \rightarrow 0} O_{t_{1} \cdots t_{k}} g(x) \\
& =\lim _{t_{1} \rightarrow 0} \cdots \lim _{t_{k} \rightarrow 0} \frac{1}{t_{1} \cdots t_{k}} \Delta_{t_{1} \xi_{1}} \cdots \Delta_{t_{k} \xi_{k}} f(x) \\
& =\frac{\partial}{\partial \xi_{1}} \cdots \frac{\partial}{\partial \xi_{k}} f(x)
\end{aligned}
$$

in $L_{p}\left(D_{2 \varepsilon}\right)$ or in $C\left(D_{2 \varepsilon}\right)$, which implies our result.

Lemma 3.2. Theorem 2.2 is valid with a constant $C(k)$ instead of 1 , that is

$$
\left\|\frac{\partial}{\partial \xi_{1}} \cdots \frac{\partial}{\partial \xi_{k}} f\right\|_{B} \leq C(k) \sup _{\xi}\left\{\left(\frac{\partial}{\partial \xi}\right)^{k} f(x) \|_{B} .\right.
$$

Proof. We have to prove the analogue of (2.4) with a constant $C(k)$ that does not depend on $\varepsilon$. This follows the Kemperman lemma ([1, p. 338]) which yields

$$
\prod_{i=1}^{k} \Delta_{h \xi_{i}}=\sum_{S \subset\{1, \ldots, k\}}(-1)^{|S|} T\left(h_{S}^{*}\right) \Delta_{h_{S}}^{k}
$$


where the sum is on all the subsets $S$ of $\{1, \ldots, k\},|S|$ is the number of elements in $S$

$$
h_{S}^{*} \equiv h \sum_{j \in S} \xi_{j} \quad \text { and } \quad h_{S} \equiv h \sum_{j \in S} j^{-1} \xi_{j} .
$$

We choose $h$ such that $2 k h<\varepsilon$ so that the transformation on the right and on the left of (3.5) is from $B$ to $B_{\varepsilon}$. The constant can now be estimated, but there is no use for it as eventually the constant 1 will be achieved.

Lemma 3.3. Under the assumptions of Theorem 2.2,

$$
\left\|\frac{\partial^{k}}{\partial \xi_{1} \cdots \partial \xi_{k}} f\right\|_{B}
$$

is continuous in $\xi_{i}$.

Proof. For our purposes it is probably sufficient to prove that

$$
\left\|\frac{\partial^{k}}{\partial \xi_{1} \cdots \partial \xi_{k}} f\right\|_{B_{c}}
$$

is continuous in $\xi_{i}$, but the result is valid as stated and may be useful in the future. We can assume the change is in $\xi_{1}$. Let $\xi_{1}^{*}-\xi_{1}=\delta \eta$ where $\eta$ is a unit vector. By earlier consideration

$$
\left\|\frac{\partial}{\partial \xi} \cdots \frac{\partial}{\partial \xi_{k}} f(x)\right\|_{B}-\left\|h^{-k} \Delta_{h \xi} \Delta_{h \xi_{2}} \Delta_{h \xi_{k}} f(x)\right\|_{B_{\varepsilon}}<\frac{\varepsilon_{1}}{3}
$$

for $\xi=\xi_{1}$ and $\xi=\xi_{1}^{*}$ for $\varepsilon$ and $h$ small enough. Using (3.4), we now write

$$
\begin{aligned}
\left\|h^{-k} \Delta_{h \xi_{1}} \cdots \Delta_{h \xi_{k}} f(x)\right\|_{B_{c}}-\| h^{-k} & \Delta_{h \xi_{1}^{*}} \Delta_{h \xi_{2}} \cdots \Delta_{h \xi_{k}} f(x) \|_{B_{\varepsilon}} \\
& \leq\left\|\delta \frac{1}{h \delta} \frac{1}{h^{k-1}} \Delta_{h \delta \eta} \Delta_{h \xi_{2}} \cdots \Delta_{h \xi_{k}} f(x)\right\|_{B_{\varepsilon}} \\
& \leq \delta\left\|O_{\delta h, h, \ldots, h} \frac{\partial}{\partial \eta} \frac{\partial}{\partial \xi_{2}} \cdots \frac{\partial}{\partial \xi_{k}} f\right\|_{B_{\varepsilon}} \\
& \leq \delta\left\|\frac{\partial}{\partial \eta} \frac{\partial}{\partial \xi_{2}} \cdots \frac{\partial}{\partial \xi_{k}} f\right\|_{B} \\
& \leq \delta C(k) \sup \left\|\frac{\partial^{k} f}{\partial \xi^{k}}\right\|_{B} .
\end{aligned}
$$

The last inequality follows from Lemma 3.2. The result is easily concluded from the above estimate.

\section{Proof of the main Result}

We are now able to prove the main result, i.e. Theorem 2.2

Proof of Theorem 2.2. The proof follows by induction on $k$. For $k=2$ we use the identity

$$
\Delta_{h \xi} \Delta_{h \eta}=\Delta_{h(\xi+\eta) / 2}^{2}-T(h \eta) \Delta_{h(\xi-\eta) / 2}^{2},
$$


set $($ for $\xi \neq \eta) \quad \xi_{1}=(\xi+\eta) /\|\xi+\eta\|=(\xi+\eta) / 2 a, \eta_{1}=(\xi-\eta) /\|\xi-\eta\|=$ $(\xi-\eta) / 2 b$ (where the norms are the Euclidean norm of $R^{d}$ ), and recall

$$
a^{2}+b^{2}=\left\|\frac{\xi+\eta}{2}\right\|^{2}+\left\|\frac{\xi-\eta}{2}\right\|^{2}=2\left(\left\|\frac{\xi}{2}\right\|^{2}+\left\|\frac{\eta}{2}\right\|^{2}\right)=1 .
$$

Using Lemma 3.1, (4.1), (4.2) and $h<\varepsilon / 4$, we have

$$
\begin{aligned}
\left\|\frac{\partial}{\partial \xi} \frac{\partial}{\partial \eta} f(x)\right\|_{B_{\varepsilon}} & =\lim _{h \rightarrow 0}\left\|h^{-2} \Delta_{h \xi} \Delta_{h \eta} f\right\|_{B_{\varepsilon}} \\
& \leq \lim _{h \rightarrow 0}\left\{a^{2}\left\|h^{-2} \Delta_{h \xi_{1}}^{2} f\right\|_{B_{\varepsilon}}+b^{2}\left\|h^{-2} \Delta_{h \eta_{1}}^{2} f\right\|_{B_{\varepsilon / 2}}\right\} \\
& \leq\left\{a^{2}\left\|\frac{\partial^{2} f}{\partial \xi_{1}^{2}}\right\|_{B}+b^{2}\left\|\frac{\partial^{2} f}{\partial \eta_{1}^{2}}\right\|_{B}\right\} \\
& \leq \sup _{\xi}\left\|\frac{\partial^{2} f}{\partial \xi^{2}}\right\|_{B},
\end{aligned}
$$

and therefore,

$$
\left\|\frac{\partial}{\partial \xi} \frac{\partial}{\partial \eta} f\right\|_{B} \leq\left\{a^{2}\left\|\frac{\partial^{2} f}{\partial \xi_{1}^{2}}\right\|_{B}+b^{2}\left\|\frac{\partial^{2} f}{\partial \eta_{1}^{2}}\right\|_{B}\right\} \leq \sup _{\xi}\left\|\frac{\partial^{2} f}{\partial \xi^{2}}\right\|_{B} .
$$

We proceed with the induction hypothesis on $0<m<k$ to obtain

$$
\begin{aligned}
I & \equiv \sup _{\xi_{1}, \ldots, \xi_{k}}\left\|\frac{\partial}{\partial \xi_{1}} \cdots \frac{\partial}{\partial \xi_{k}} f\right\|_{B} \\
& \leq \sup _{\xi, \xi_{m+1}, \ldots, \xi_{k}}\left\|\left(\frac{\partial}{\partial \xi}\right)^{m} \frac{\partial}{\partial \xi_{m+1}} \cdots \frac{\partial}{\partial \xi_{k}} f\right\|_{B} \\
& \leq \sup _{\xi, \eta}\left\|\left(\frac{\partial}{\partial \xi}\right)^{m}\left(\frac{\partial}{\partial \eta}\right)^{k-m} f\right\|_{B} \\
& \equiv I(m) \leq I .
\end{aligned}
$$

We observe, using Lemma 3.3, that maximum can replace supremum in both $I(m)$ and $I$. We need to show that for some direction $\zeta$

$$
I=I(m)=\left\|\frac{\partial^{k}}{\partial \zeta^{k}} f\right\|_{B}
$$

Given $0<m<k$, we have for some $\xi$ and $\eta$

$$
I=I(m)=\left\|\frac{\partial^{m}}{\partial \xi^{m}} \frac{\partial^{k-m}}{\partial \eta^{k-m}} f\right\|_{B} .
$$

If $\xi=\eta$, the theorem is proved, and therefore, we assume $\xi \neq \eta$. If $k$ is even, we choose $m$ so that $2 m=k$, and using the first inequality in (4.3) repeatedly, 
we have

$$
\begin{aligned}
I & =I\left(\frac{k}{2}\right)=\left\|\frac{\partial^{m}}{\partial \xi^{m}} \frac{\partial^{m}}{\partial \eta^{m}} f\right\|_{B} \\
& \leq \sum_{l=0}^{m}\left(\begin{array}{c}
m \\
l
\end{array}\right) a^{2 l} b^{2 m-2 l}\left\|\frac{\partial^{2 l}}{\partial \xi_{1}^{2 l}} \frac{\partial^{2 m-2 l}}{\partial \eta_{1}^{2 m-2 l}} f\right\|_{B} \\
& \leq a^{2 m}\left\|\frac{\partial^{2 m}}{\partial \xi_{1}^{2 m}} f\right\|_{B}+\left(\sum_{l=0}^{m-1}\left(\begin{array}{c}
m \\
l
\end{array}\right) a^{2 l} b^{2 m-2 l}\right) I,
\end{aligned}
$$

and as $a^{2}+b^{2}=1$, we have

$$
\sum_{l=0}^{m-1}\left(\begin{array}{c}
m \\
l
\end{array}\right) a^{2 l} b^{2 m-2 l}=1-a^{2 m},
$$

and therefore,

$$
I=\left\|\frac{\partial^{2 m}}{\partial \xi_{1}^{2 m}} f\right\|_{B}
$$

For odd $k$ we construct a sequence $\left\{m_{i}, \xi_{i}, \eta_{i}\right\}$ such that $0<m_{i}<k$,

$$
I=I\left(m_{i}\right)=\left\|\frac{\partial^{m_{i}}}{\partial \xi_{i}^{m_{i}}} \frac{\partial^{k-m_{i}}}{\partial \eta_{i}^{k-m_{i}}} f\right\|_{B}
$$

and the angle between $\xi_{i+1}$ and $\eta_{i+1}$ is half the angle between $\xi_{i}$ and $\eta_{i}$. For $m_{i}<k / 2$ we choose $\xi_{i+1}=\left(\xi_{i}+\eta_{i}\right) /\left\|\xi_{i}+\eta_{i}\right\|, \eta_{i+1}=\eta_{i}$ and $m_{i+1}=2 m_{i}$, while for $m_{i}>k / 2$ we choose $\xi_{i+1}=\xi_{i}, \eta_{i+1}=\left(\xi_{i}+\eta_{i}\right) /\left\|\xi_{i}+\eta_{i}\right\|$ and $k-m_{i+1}=2\left(k-m_{i}\right)$. To show that (4.4) is valid for $i+1$, we write for $m_{i}<k / 2$

$$
\zeta \equiv\left(\xi_{i}-\eta_{i}\right) /\left\|\xi_{i}-\eta_{i}\right\| \text { and } a_{i} \equiv \frac{1}{2}\left\|\xi_{i}+\eta_{i}\right\|
$$

and hence

$$
\begin{aligned}
I=I\left(m_{i}\right) & \leq \sum_{l=0}^{m_{i}}\left(\begin{array}{c}
m_{i} \\
l
\end{array}\right) a_{i}^{2 l}\left(1-a_{i}^{2}\right)^{m_{i}-l}\left\|\frac{\partial^{2 l}}{\partial \xi_{i+1}^{2 l}} \frac{\partial^{2 m_{i}-2 l}}{\partial \zeta^{2 m_{i}-2 l}} \frac{\partial^{k-2 m_{i}}}{\partial \eta_{i+1}^{k-2 m_{i}}} f\right\|_{B} \\
& \leq a_{i}^{2 m_{i}}\left\|\frac{\partial^{2 m_{i}}}{\partial \xi_{i+1}^{2 m_{i}}} \frac{\partial^{k-2 m_{i}}}{\partial \eta_{i+1}^{k-2 m_{i}}} f\right\|_{B}+\left(1-a_{i}^{2 m_{i}}\right) I .
\end{aligned}
$$

The case $m_{i}>k / 2$ is symmetric. As $\left\|\xi_{i}-\eta_{i}\right\| \rightarrow 0$, Lemma 3.3 yields our theorem.

\section{EXTENSIONS TO OTHER FUNCTION SPACES AND OTHER DOMAINS}

As Theorem 2.2 is valid for $C(D)$ for all open sets $D$, it is valid for $C\left(R^{d}\right)$. From this we may deduce the result to a Banach space of functions or generalized functions on $R^{d}$ for which translation is an isometry, that is

$$
\|T(y) f(\cdot)\|_{B}=\|f(\cdot+y)\|_{B}=\|f(\cdot)\|_{B} .
$$


Translation is called strongly, weakly or weakly ${ }^{*}$ continuous if $T(y) f-f \rightarrow 0$ (as $\|y\| \rightarrow 0$ ) in the strong, weak or weak ${ }^{*}$ topology, respectively (the last only when $X^{*}=B$, for some Banach space $X$ ).

Theorem 5.1. Suppose $B$ is a Banach space of functions or generalized functions on $R^{d}$ for which translation is a strongly, weakly or weakly ${ }^{*}$ continuous isometry. Then $\partial^{k} f / \partial \xi^{k} \in B$ for all $\xi$ implies $\partial^{k} f /\left(\partial \xi_{1} \cdots \partial \xi_{k}\right) \in B$ where derivatives are taken in the strong, weak or weak ${ }^{*}$ sense, respectively, and

$$
\left\|\frac{\partial^{k}}{\partial \xi_{1} \cdots \partial \xi_{k}} f(\cdot)\right\|_{B} \leq \sup _{\xi}\left\|\frac{\partial^{k}}{\partial \xi^{k}} f(\cdot)\right\|_{B} .
$$

Proof. We define

$$
F(x)=\langle f(x+\cdot), g(\cdot)\rangle
$$

for $g \in B^{*}$ in case the strong or weak result is proved, and for $g \in X, X^{*}=$ $B$ in case the weak ${ }^{*}$ result is proved. We set $\|g\|_{B^{*}}=1$ or $\|g\|_{X}=1$, respectively, and write

$$
\left\|\frac{\partial^{k} F(\cdot)}{\partial \xi_{1} \cdots \partial \xi_{k}}\right\|_{C\left(R^{d}\right)} \leq \sup _{\xi}\left\|\frac{\partial^{k} F(\cdot)}{\partial \xi^{k}}\right\|_{C\left(R^{d}\right)} \leq \sup _{\xi}\left\|\frac{\partial^{k} f(\cdot)}{\partial \xi^{k}}\right\|_{B} .
$$

As

is given for any $g$,

$$
\frac{\partial^{k}}{\partial \xi_{1} \cdots \partial \xi_{k}} F(0)
$$

exists and we may choose $g_{\varepsilon}$ such that

$$
\frac{\partial^{k} f}{\partial \xi_{1} \cdots \partial \xi_{k}}
$$

$$
\begin{aligned}
\left\|\frac{\partial^{k}}{\partial \xi_{1} \cdots \partial \xi_{k}} F\right\|_{C\left(R^{d}\right)} & \geq\left\|\frac{\partial^{k}}{\partial \xi_{1} \cdots \partial \xi_{k}} F(0)\right\| \\
& =\left|\left\langle\frac{\partial^{k}}{\partial \xi_{1} \cdots \partial \xi_{k}} f(\cdot), g_{\varepsilon}(\cdot)\right\rangle\right| \\
& \geq\left\|\frac{\partial^{k}}{\partial \xi_{1} \cdots \partial \xi_{k}} f\right\|_{B}-\varepsilon
\end{aligned}
$$

with any $\varepsilon>0$.

Remark 5.2. Theorem 5.1 applies to $L_{\infty}\left(R^{d}\right)$ as translation is weakly ${ }^{*}$ continuous isometry in that space.

Remark 5.3. For an open set $\Omega$ for which a function $f \in W_{p}^{r}(\Omega)$ can be extended to $F \in W_{p}^{r}\left(R^{d}\right)$, that is $F(x)=f(x)$ for $x \in \Omega$ and for which $m(\partial \Omega)=0$, we have

$$
\left\|\frac{\partial^{k}}{\partial \xi_{1} \cdots \partial \xi_{k}} F\right\|_{L_{p}(\bar{\Omega})} \leq \sup _{\xi}\left\|\frac{\partial^{k} f}{\partial \xi^{k}}\right\|_{L_{p}(\Omega)}, \quad 0<k \leq r .
$$


The same is valid for the norms $C(\bar{\Omega})$ and $C(\Omega)$ if $\Omega$ is such that $f \in C^{r}(\Omega)$ can be extended to $F \in C^{r}\left(R^{d}\right)$. Such extensions are discussed extensively elsewhere (see [3] and [4]).

Remark 5.4. In Theorem 2.2, $L_{p}(\Omega)$ can be replaced by any Banach space of functions on $\Omega, B(\Omega)$ which satisfy:

$$
\begin{gathered}
|f(x)| \geq|g(x)| \text { for } x \in \Omega \text { implies }\|f\|_{B(\Omega)} \geq\|g\|_{B(\Omega)}, \\
\left\|O_{t_{1} \cdots t_{k}} f\right\|_{B\left(\Omega_{\varepsilon}\right)} \leq\|f\|_{B(\Omega)} \text { for } t_{i} \text { small enough }
\end{gathered}
$$

and

$$
\|f\|_{B\left(\Omega_{\varepsilon}\right)} \leq M \text { implies } \lim _{\varepsilon \rightarrow 0^{+}}\|f\|_{B\left(\Omega_{\varepsilon}\right)}=\|f\|_{B(\Omega)} .
$$

It is easy to see that properties (1), (2) and (3) are satisfied by many spaces and are not particular to $L_{p}$.

Remark 5.5. As $\left(R_{+}^{d}\right)^{0}=\Omega$ satisfies the condition in Remark 5.3, (5.3) is valid for $C\left(R_{+}^{d}\right)$, and therefore, the method of Theorem 5.1 will imply validity for any Banach spaces on $R_{+}^{d}$ for which translation $T(y)$ for $y \in R_{+}^{d}$ is a contraction which is strongly, weakly or weakly ${ }^{*}$ continuous.

\section{REFERENCES}

1. C. Bennett and R. Sharpley, Interpolation of operators, Academic Press, 1988.

2. Z. Ditzian, Multivariate Landau-Kolmogorov-type inequality, Math. Proc. Cambridge Philos. Soc. 105 (1989) 335-350.

3. R. Sharpley, Cone conditions and the modulus of continuity, Canad. Math. Soc. Conf. Proc. 3 (1983), 341-351.

4. E. M. Stein, Singular integrals and differentiability properties of functions, Princeton University Press, Princeton, New Jersey, 1970.

Department of Mathematics, University of Alberta, Edmonton, Alberta, T6G 2G1, CANADA 